Original Article

\title{
The UV-Vis spectrum of antioxidant complex from frozen-dried egg white protein and tomato extract
}

\author{
Siti Imama Khoiriyah, Mohammad Mabrur, Sutiman Bambang Sumitro, Sri Widyarti* \\ Department of Biology, Faculty of Mathematics and Natural Sciences, Brawijaya University, Jalan Veteran, Malang, East Java 65145, Indonesia
}

Abstract

In this study, we want to characterize egg white protein-tomato extract complex based on the UV-Vis spectrum. The formulation of antioxidant complex Non-Freeze-drying (NFD) was made at a ratio of 1:10 (v/v) while the Freeze-drying (FD) sample was made at a ratio of 1:2 (v/v). The results showed that the absorption peak of FD complex profile value was lower than the single ingredient, while the tomato extract was higher than the complex profile. On the contrary, the NFD complex showed a higher peak absorption value than to every single ingredient. Based on each UV-Vis spectrum profile, the tomato extract profile shifted the absorption peak, and the absorbance value of the FD sample was changing higher than the NFD sample, while the egg white protein spectrum profile did not change significantly in the FD and NFD samples. The absorption peak of the complex is higher than the single ingredient indicated that the characterization of the complex had been formed. In the NFD complex profile, the absorption peaks formed at the wavelength regions of $230 \mathrm{~nm}$ and $280 \mathrm{~nm}$ with absorbance values of 2.51 and 0.76 and its number has higher than those of the FD complex with absorbance values of 2.47 and 1.82 at wavelengths of 220 and $265 \mathrm{~nm}$.

Keywords: Antioxidant complex, egg white protein, freeze-drying, UV-vis spectrum.

Received: 25 April 2020 Revised: 13 June 2020 Accepted: 14 June 2020

\section{Introduction}

Tomato is one of the agricultural product which contains the essential value of nutritional food (Temitope et al., 2009). In general, tomatoes can be processed into various products or consumed freshly (ArkoubDjermoune et al., 2019). Regarding the nutrition need, many people are initially realized that "Functional Food" is necessary for the health benefit (Pinela et al., 2016). Tomatoes contain several types of natural antioxidants, including vitamin E, ascorbic acid, carotenoids, flavonoids, and phenolic compounds. Lycopene is a type of carotenoid in tomatoes which regarded as an antioxidant activitor. Compared to another natural antioxidant, such as $\beta$-carotene, $\alpha$-carotene, vitamin $C$ and ascorbic acid, the content of lycopene in tomatoes per 100 grams is $2,573 \mu \mathrm{g}$ (Haroon, 2014).

Lycopene acts as a free radical scavenger, which reduces singlet oxygen and peroxyl radicals (Osterlie \& Lerfall, 2004). The combination of lycopene with vitamins $\mathrm{C}, \mathrm{E}$ and $\beta$-carotene give a more robust scavenger activity than that of a single antioxidant (KinWeng Kong et al., 2010). To keep in the stable state, complex lycopene is expected to have a stronger antioxidant activity once reacted with other molecules. Lycopene has been recorded having 11 conjugated hydrophobic double bonds. In addition, these compounds are sensitive to light, oxygen and acids and metals,

\footnotetext{
* Corresponding Author:

Sri Widyarti

Department of Biology, Faculty of Mathematics and Natural Science, Brawijaya University, Jalan Veteran, Malang, East Java 65145, Indonesia

Phone: +62 341551611

Fax: $+62341-554403$

e-mail: swid@ub.ac.ic
}

Moreover, this influences the compounds being unstable (Shi, 2000). The protein structure in the egg white contains a polymer that protects the hydrophobicity of antioxidants in tomatoes. Specifically, the egg white component contains 54\% ovalbumin supplemented with six cysteine residues. Two of these residues are involved in disulfide bonds, while the other four residues belong to the free sulfhydryl group (Guha et al., 2018). In the cutting-edge technology, the egg white protein as a polymer has not been widely studied for the drug delivery system. Hence, it is desirable to observe. Albumin application as drug delivery concept is one of the cuttingedge of knowledge that promises as a commercial product to be developed (Sethi et al., 2013).

In this study, the formulation of the antioxidant complex was carried out by freeze-drying (FD) and nonfreeze-drying (NFD) to compare the effect of changing spectrum profiles. Freeze-dry technology is needed as a complex formulation preparation and used as an alternative candidate for drug delivery system, resulting in an increase of antioxidant stability supplemented with egg white protein as an encapsulation compound (Chen \& Wang, 2007). The freeze-dry process can produce particles with a small size and a longer shelf life with a low cost compared to supercritical fluid extraction (Nireesha et al., 2013).

This research formulates antioxidant complex based on a crude extract of tomatoes with egg white protein to enhance the bioavailability of egg whites that commonly consumed, and chosen as an alternative for drug delivery system due to an essential component of egg whites such as ovalbumin, that known as a copolymer with hydrophilic and hydrophobic structures, and their 
capability to stabilize an encapsulation of the target compound (Lohcharoenkal et al., 2014).

Moreover, antioxidant complexs are expected to increase ovalbumin as a scavenger agent by the complex formation between egg white protein and tomato extract. To describe the characterization of the antioxidant complexes that processed by FD and NFD, then it was further studied through UV-Vis spectrophotometer instrument. The spectrum profile analysis of FD and NFD samples were tested as a preliminary study to observe the interaction between tomato extract and egg white protein. The principal of UV-Vis spectrophotometers is based on the material absorption by light that depends on the amount of light and wavelength. The electromagnetic radiation used in spectrophotometric measurements is divided into two ultraviolet (UV, 190-400 nm) and visible regions (VIS, 400-800 nm) (Kumar, 2006). In addition, spectroscopic is one of significant and straight forward methods used to analyze structural changes and explore complex formation (Khoirunnisa et al., 2019).

\section{Methods}

\section{Materials}

Chicken eggs were obtained from the University of Muhammadiyah Malang farm, while the tomatoes were obtained from the traditional market at Dinoyo-Malang. Phosphate-Buffer Saline (PBS) (pH 7.4) was prepared by dissolving 8 gr NaCl; $200 \mathrm{mg} \mathrm{KCl} ; 1.44$ gr Na${ }_{2} \mathrm{HPO}_{4} ; 240$ mg $\mathrm{KH}_{2} \mathrm{PO}_{4}$ in $1 \mathrm{~L}$ distilled water.

\section{Sample Preparation}

The fresh tomatoes were processed using a juicer for NFD preparation. Then, the extract was added a distilled water 1:100 (v/v) following centrifugation at $4000 \mathrm{rpm}$, $10 \mathrm{~min}$ at $20^{\circ} \mathrm{C}$. Then the supernatant was collected. The egg white protein was separated manually from the yolk, and then egg white protein was dissolved in PBS 1:100 $(\mathrm{v} / \mathrm{v})$.

\section{Preparation of the Non-Freeze-drying (NFD) complex}

The complex NFD, mixed egg white protein and tomato extract 1:10 (v/v) were homogenized with a magnetic stirrer plate for $60 \mathrm{~min}$ at room temperature and incubated overnight at $4^{\circ} \mathrm{C}$.

\section{Preparation of the Freeze-drying (FD) complex}

The sample was frozen in the freezer for $24 \mathrm{~h}$ following with freeze-drying process. The FD tomato extract was obtained in pasta form, while egg white protein was obtained in the powder form. A total of 52.5 $\mathrm{mg} / \mathrm{ml}$ of freeze-dried tomato was dissolved in distilled water. Freeze-dried egg white protein was prepared in PBS ( $\mathrm{pH} \mathrm{7.4)} \mathrm{with} \mathrm{a} \mathrm{final} \mathrm{concentration} \mathrm{of} 0.37 \mathrm{mg} / \mathrm{ml}$. The FD complex was formulated with a ratio 1:2 (v/v) (egg white:tomato extract), then this complex was homogenized with a magnetic stirrer for $60 \mathrm{~min}$ at room temperature.

\section{UV-Vis spectrum absorbance}

Characterization of single and complex compounds was quantified using a Thermo spectrophotometer
(Genesys 10 UV) (Rochester, USA). The spectrophotometer was set up in the survey scan mode $(\boldsymbol{\lambda})$ 200-600 nm.

\section{Results}

The characteristics of antioxidant complexs, tomato extract and egg white protein, were analyzed using the UV-Vis spectrum (Figure 1-2). Figure 1 showed the absorption peak of single ingredient (egg white protein and tomato extract) NFD and FD, while figure $2 a-b$ showed the absorption peak of antioxidant complex NFD and FD. The changes in absorption peaks NFD and FD samples in both single ingredient and antioxidant complex were observed in the formed profile. The results showed that the single and antioxidant complex, NFD and FD samples, had shifted absorption peaks (Table 1).

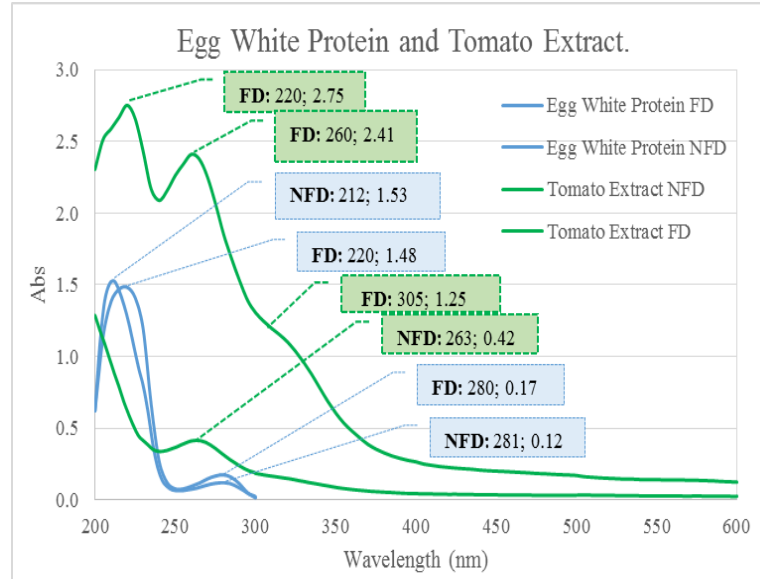

Figure 1. Spectrum profiles FD and NFD of egg white protein and tomato extract.

The complex formulations, FD and NFD, showed a different profile as depicted in the absorption peak values. The absorption peak value in the FD complex was lower than the tomato extract, while the absorption peak value in the NFD complex was higher than the single NFD sample. Based on the profile of UV-Vis spectrum results (Figure 2a-b), it was hypothesized that the NFD complex (Figure 2b) formed the antioxidant complex, which indicated by the high value of absorption peak.

The shifting of absorption peak from each sample was followed by changing an absorbance values of both the NFD and FD processes. The profile of NFD egg white protein was detected at 212 and $281 \mathrm{~nm}$, which showed a shifting peak at the absorption peak of FD samples at 220 $\mathrm{nm}$ and $280 \mathrm{~nm}$. While the profile of NFD tomato extracts dissolved in distilled water detected two peaks at 200 and $263 \mathrm{~nm}$ which showed a shift of absorption peak in the tomato extract FD at 220, 260 and $305 \mathrm{~nm}$ in the ultraviolet region. Unlike the case with the characteristics of a single ingredient, the characteristics of absorption peaks NFD and FD complexes formed two different new peaks with absorbance values around 2.5 at each absorption peak.

The absorption peak NFD samples were detected at 230 and $280 \mathrm{~nm}$, while the FD samples showed a shift in the absorption peaks of 220 and $265 \mathrm{~nm}$. In addition, the 
formulation of the complex profiles has been presumed that tomato extract could form complexes with egg white protein, which was seen in changes in the profile of each

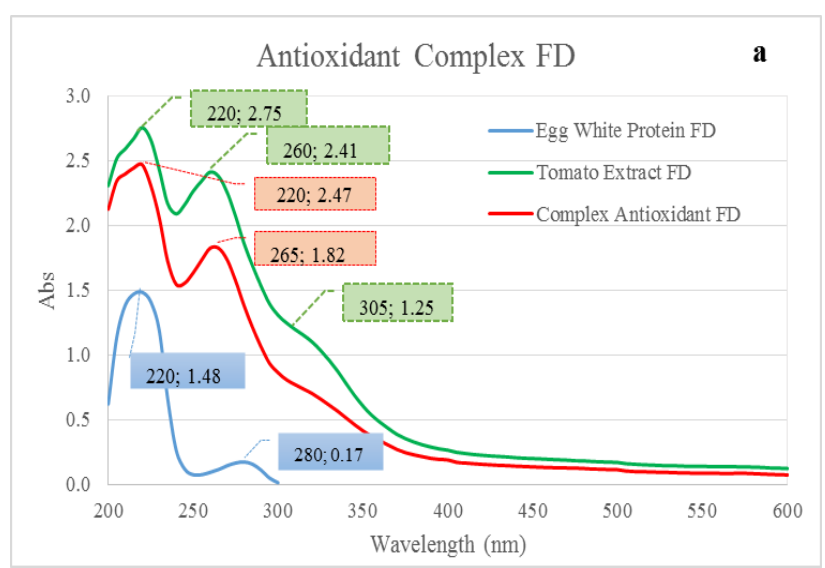

single ingredient followed by changes in the absorption peak of the complex profile.

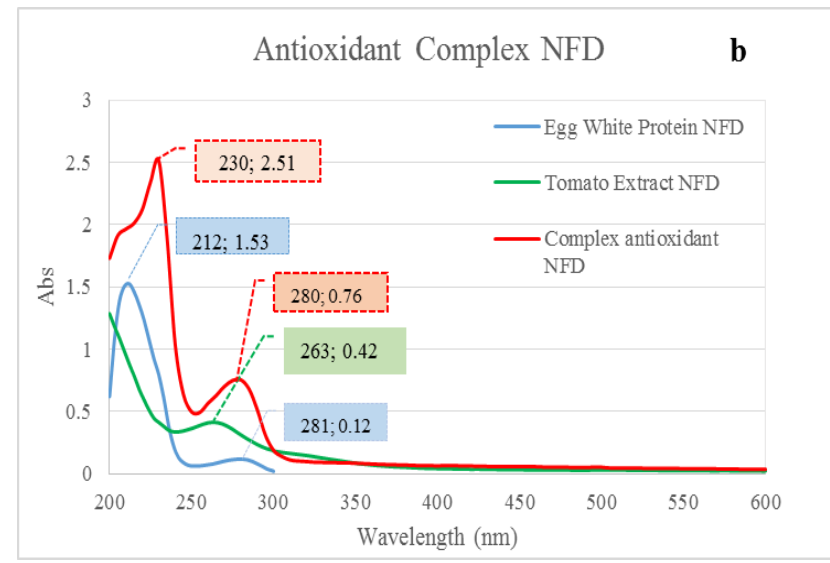

Figure 2. Antioxidant complex (a) Spectrum profile FD complex with egg white protein and tomato extract FD. (b) Spectrum profile NFD complex with egg white protein and tomato extract NFD.

Table 1. The absorption peak of UV-Vis spectrum profile

\begin{tabular}{ccc}
\hline Sample & Wavelength $(\mathbf{n m})$ & Absorbance \\
\hline NFD Tomato & 263 & 0.42 \\
& 220 & 2.75 \\
Tomato FD & 260 & 2.41 \\
& 305 & 1.25 \\
\multirow{2}{*}{ NFD Egg White } & 212 & 1.53 \\
& 281 & 0.12 \\
Egg White FD & 220 & 1.48 \\
& 280 & 0.17 \\
NFD Complex & 230 & 2.51 \\
& 280 & 0.76 \\
FD Complex & 220 & 2.47 \\
& 265 & 1.82 \\
\hline
\end{tabular}

\section{Discussion}

The absorbance value of the FD complex sample in the spectrum profile has decreased, while the NFD complex sample has increased with the highest absorption peak. The difference in the absorption area indicates the difference in chromophore groups that cause the differences ability to absorb UV light (Kaijanen et al., 2015). Neldawati et al. (2013) showed that the absorbance is a comparison of light intensity that comes with absorbed light intensity. The absorbance value of the sample depends on the concentration contained in the substance, the higher concentration of the substance, the higher the absorbance value obtained in the solution Chromophores and auxochromes influence the spectrum profile. A chromophore is a functional group that causes the absorption energy from ultraviolet radiation and visible light, apart from the chromophore can be influenced by auxochromes and affect the color intensity of a molecule (Shah et al., 2015).

In this study, the spectrum profile has a new peak character that was formed by the combination of egg white protein and tomato extract, in which the wavelength shifts from the peak of a single ingredient was observed. This result was hypothesized that the complex of each

process occurred by the formation of a spectrum profile through the measurement of UV-Vis spectrophotometer. In the previous studies, the formation of the $\beta$ cyclodextrin-lycopene complex, measured using a UVVis spectrophotometer, showed a shift in the absorption peaks and a higher absorbance in the complex compounds (Wang et al., 2019). They were shifting the absorption peak of NFD samples with FD samples in the direction of lower wavelength or hypsochromic shift (blue shift). The hypsochromic shift is the absorption peak shifting towards shorter wavelengths or higher energy (Kumar, 2006). This can be influenced by the presence of the attachment auxochromes to the chromophore and can affect the color intensity (Shah et al., 2015). Physically, the material changes from the NFD to FD sample shows that the FD sample formed a bright color when dissolved with distilled water while the NFD sample formed slightly more orange color. The color change is predicted as an effect of the absorption peaks shift at lower wavelengths with higher energy due to direct light transmission.

The different character of the complex formed shows that the absorbance peak value of FD complex is lower 
than the tomato extract and higher than the egg white protein. Unlike the NFD complex profile, that shows the highest absorption peak value compared to all NFD single ingredients. Sambasevam et al. (2013) and Wang et al. (2007) reported that the interaction of the complex, $\beta$ cyclodextrin with azomethine and $\beta$-cyclodextrin with orange-G, showed a higher absorption peak complex than to a single ingredient. Hence, the absorption peak value of NFD complex samples can be assumed to form complexes between egg white protein and tomatoes extract. The existence of complexes that measured using a $\mathrm{UV}-\mathrm{Vis}$ spectrophotometer needs a further investigation to confirm their complex formation and composition using Fourier Transform-Infrared (FT-IR) spectroscopy.

The formulation of egg white-tomato extracts is one of the simple techniques to obtain more stable antioxidant properties in tomatoes. The use of egg white protein as a polymer drug delivery alternative is based on the ovalbumin component, and ovalbumin is known as copolymers with hydrophilic and hydrophobic structures that able to encapsulate a target compound with a stable condition (Lohcharoenkal et al., 2014). Moreover, the local wisdom of Indonesian people believed that egg white protein has functional food and medicine value. Egg white protein, one type of animal protein, has great potential as an antioxidant source. Nevertheless, the corresponding research has remained a lack of observation compared to vegetable antioxidants. More than that, egg white protein contains abundant amino acids that essential for health value such as histidine, isoleucine, leucine, lysine, methionine, phenylalanine, threonine, tryptophan, and valine (Mann, 2017). Besides the presence of thiol groups, aromatic side chains (tryptophan, tyrosine, and phenylalanine) and imidazole in the histidine of egg white protein has been exploited as an important feature for antioxidant activity (Nirmalaratne $\& \mathrm{Wu}, 2015)$.

In their application, instead of a scavenger of a free radical, egg white protein has been known as a polymer (Lohcharoenkal et al., 2014). Hence, egg white protein can be exploited as an encapsulation agent or a drug delivery system by binding to the hydrophobic amino acids. The alkyl group of lycopene is known to form hydrophobic bonds with five amino acid residues including valine, arginine, proline and leucine. Moreover, lycopene also able to interacts with polar/hydrophilic amino acids, including arginine in ovalbumin. Some studies also reported regarding molecular dynamics of ovalbumin from egg white protein with the antioxidant lycopene, that lycopene will insert and interact with a hydrophobic region of ovalbumin and binds with the hydrophobic region of ovalbumin non-polar amino acid residue. The formation of ovalbumin complex with lycopene doesn't interfere with ovalbumin characters as an antioxidant, both compounds don't bind to cysteine amino acid residues and don't have a same binding site as ovalbumin ligands in the form of NAG (N-Acetyl-DGlucosamine) (Lestari, 2019 ).

The use of egg white protein as a drug delivery system is not widely known because some studies focused on using cyclodextrin from cyclic oligosaccharides (Santos et al., 2017). Polyakov et al. (2006) showed that the cyclodextrin as an encapsulation of bioactive components when in a single molecule state is known to increase stability, solubility and antioxidant activity of guest compounds in the polymer core. Based on in vitro radical peroxide antioxidant activity testing, the cyclodextrincarotenoid complex showed an increase in carotenoid stability and solubility, but there was no increase in antioxidant activity. Aside from aiming as a hydrophobic antioxidant stability, complex formation is also expected to increase antioxidant activity stronger than the single form. So that is one of the reasons underlying the use of egg white protein as a drug delivery system that is better than cyclodextrin. Egg white protein acts as a transporter also has the ability as a scavenger of free radicals in the presence of free suflhidril groups (thiol). The presence of thiol groups and aromatic amino acid side chains (tryptophan, tyrosine, and phenylalanine) and imidazoler in histidine are recognized as important structural properties in antioxidant activity (Nirmalaratne \& $\mathrm{Wu}$, 2015). Hence, the use of egg white as a drug delivery system and transporter is better than cyclodextrin.

The formation of complex, FD and NFD, was quantified by a UV-Vis spectrophotometer and showed a new profile with two different absorption peaks from each FD and NFD (compound profile of tomato extract and egg white). The FD sample complex formed has a lower absorbance (2.47) than the tomato extract, and higher than the egg white protein (1.82). While the complex profile of NFD samples has a higher absorbance (2.51) than the tomato extract and egg white protein. The NFD profile of tomato extract (Tabel 1) have an absorption peak at 263 $\mathrm{nm}$ with an absorbance value of 0.42 . Unlike the FD profile of tomato extract which showed 3 absorption peaks of $2.75 ; 220 \mathrm{~nm}, 2.41$ at $260 \mathrm{~nm}$; and 1.25 at 305 $\mathrm{nm}$, respectively. Whereas egg white protein profile of NFD and FD each formed 2 absorption peaks. The absorption peak in NFD egg white protein samples (at 212 and $281 \mathrm{~nm}$ ) has an absorbance value of 1.53 and 0.12 , respectively, and the absorption peak in FD egg white protein samples (at 220 and $280 \mathrm{~nm}$ ) have an absorbance value of 1.48 and 0.17 , respectively. Furthermore, the NFD complex profile detected 2 absorption peaks (at 230 and $280 \mathrm{~nm}$ ) with absorbance values of 2.51 and 0.76 , respectively, whereas FD complex have 2 absorption peaks (at 220 and $265 \mathrm{~nm}$ ) with absorbance values of 2.47 and 1.82, respectively. Based on the previous studies (Lestari, 2019) showed that the interaction of ovalbumin, a major component of egg white protein, with lycopene is one of the antioxidants in tomatoes, that capable of forming complexes that bind to hydrophobic regions with residual non-polar ovalbumin amino acids

In this research, the extraction a single ingredient of lycopene or ovalbumin was not performed, but the material used, egg white protein, had major ovalbumin component of 54\% (Hoppe, 2010), while based on the HPLC analysis (data not shown) freeze-drying tomatoes dissolved with distilled water had lycopene content of $5.65 \mu \mathrm{g} / \mathrm{ml}$. Among the commonly drying methods, freeze-drying is considered as one of the current methods having high-quality values, due to its benefits such as able to produce ingredients with high porosity, good 
nutritional quality, texture, aroma, taste, and colours with good capacity. However, besides the advantages, the FD process remained some drawbacks such as expensive and time-consuming (Muñoz-López et al., 2018). The NFD process cannot be preserved for a long time, as well as less effective and efficient materials. In this study, an antioxidant complex, egg white protein and tomato extract from the NFD and FD processes, was quantified using a UV-Vis spectrophotometer. All processes are carried out without the extraction of compounds, to obtain all of the compounds in tomatoes that essential including lycopene, flavonoid and phenolic. Bhowmik et al. (2012) showed that tomatoes have a potential candidate as antiinflammatory and antithrombotic. In the previous studies showed that consuming tomatoes reduced the risk of cancer and other diseases such as cardiovascular, osteoporosis, skin problems and cognitive dysfunction. In this study crude extract tomato dissolved with distilled water did not show a lycopene profile (Figure 1). The formation peak is recognized as the interaction of complex molecules of tomatoes with electromagnetic waves. However, the egg white protein has a different spectrum profile (Figure 1) which shows the spectrum of proteins is soluble in PBS ( $\mathrm{pH} \mathrm{7.4).} \mathrm{Albumin} \mathrm{is} \mathrm{a} \mathrm{globular}$ protein that is soluble in water and salt but can be denaturated by heat ( $\mathrm{Li}$ et al., 2017). Besides, the egg white component is composed of the hydrophobic amino acids such as isoleucine, leucine, methionine, and valine (Mann, 2017).

Freeze-drying is one of the effective methods for compound preparation compared to other methods such as evaporation and co-participation (Wang et al., 2019). Each of the FD and NFD processes has advantages and disadvantages. In this study, the freeze-drying method was chosen due to its essential advantages, that able to increase the stability of samples (Nail \& Gatlin, 1992). Freeze-drying is an indispensable technology in formulating air materials nano-scale, hence, in the last few decades, many studies have begun to apply the technology. Freeze-drying consists of two stages, sublimation (first drying) and desorption (second drying). The freeze-drying process has a cheap process compared to supercritical fluid extraction, extend the storage period of the material and maintain the stability of the material. (Chen \& Wang, 2007; Nireesha et al., 2013)

In conclusion, The UV-Vis spectrum profile of NFD complex showed a higher peak absorption value compared to the single ingredient while the FD complex profile had lower peak absorption value compared to the single ingredient (tomato extract). The peak value of complex samples is higher than a single ingredient can be indicated as complex formation. In addition, the confirmation of the complex formation and the composition of their functional groups is urgently necessary using the FT-IR (Fourier Transform-Infrared) spectroscopy.

\section{Acknowledgments}

The research was funded by DPP-SPP FY 2019 Faculty of Mathematic and Natural Sciences, Brawijaya University.

\section{References}

Arkoub-Djermoune, L., Bellili, S., Khenouce, L., Benmeziane, F., Madani, K., \& Makhlouf, L. (2019). Effect of domestic cooking on physicochemical parameters, phytochemicals and antioxidant properties of Algerian tomato (Solanum Lycopersicum L. Var. Marmande). Journal of Food Technology Research, 6(1), 1-17. doi:10.18488/journal.58.2019.61.1.17

Bhowmik, D., Kumar, K., Paswan, S., \& Srivastava, S. (2012). TomatoA Natural Medicine and Its Health Benefits. Journal of Pharmacognosy and Phytochemistry, 1(1), 24-36.

Chen, G., \& Wang, W. 2007. Role of freeze-drying in nanotechnology. $\begin{array}{llll}\text { Drying Technology, 29-35. } & \end{array}$ doi:10.1080/07373930601161179

Guha, S., Majumder, K., \& Mine, Y. (2018). Egg Proteins. Department of Food Science and Technology, University of NebraskaLincoln, NE, United States; and Department of Food Science, University of Guelph, Guelph, ON, Canada. Elsevier Inc.

Haroon, S. (2014). Extraction of lycopene from tomato paste and its immobilization for controlled release. (Master), Masters of Science in Materials \& Processing Engineering. University of Waikato.

Hoppe, A., (2010). Examination of egg white protein s and effects of high pressure on select physical and functional properties. (Master), Major: Food Science \& Technology Under the Supervision of Professor Michael G, Zeece Nebraska, Nebraska.

Li, S., Cao, Y., \& Geng, F. (2017). Genome-wide identification and comparative analysis of albumin family in vertebrates. Evolutionary Bioinformatics, 13, 1-6. doi:10.1177/1176934317716089

Kaijanen, L., Paakkunainen, M., Pietarinen, S., Jernstrom, E., \& SatuPia, R. (2015). Ultraviolet detection of monosaccharides: multiple wavelength strategy to evaluate results after capillary zone electrophoretic separation. International Journal of Electrochemical Science, 10, 2950-2961.

Kong, K. W., Khoo, H. E., Prasad, K. N., Ismail, A., Tan, C. P., \& Rajab, N. F. (2010). Revealing the power of the natural red pigment lycopene. Molecules, 15(2), 959-987. doi:10.3390/molecules15020959

Khoirunnisa, W., Nur, M. I., Widyarti, S., Permana, S., \& Sumitro, S. B. (2019). Physiologic glycated-bovine serum albumin determination using spectrum-UV. Journal of Physics: Conference Series, 1241, 1-7. doi:10.1088/1742-6596/1241/1/012003

Kumar, S. (2006). Organic chemistry spectroscopy of organic compounds. Dept. of Chemistry, Guru Nanak Dev University. Amritsar -143005.

Lestari, E. (2019). Molecular dynamic study of ovalbumin complex with lycopene, lycopene-Fe $e^{3+}$, lycopene- $\mathrm{Cu}^{2+}$ at acidic $\mathrm{pH}$. (Assay), Department of Biology, Faculty of Mathematics and Natural Science, Brawijaya University, Malang.

Lohcharoenkal, W., L. Wang, YC, Chen, \& Y. Rojanasakul. (2014) Protein nanoparticles as drug devery carriers for cancer therapy. BioMed Research International, 1(1), 1-12.

Mann, K. (2017). Proteomic of egg white. Proteomic in Food Science, 6, 261-276.

Muñoz-López, C., Urrea-Garciaa, G. R., Jiménez-Fernandezb, M., Rodríguez-Jiménesc, G. C., \& Luna-Solano, G. (2018). Effect of drying methods on the physicochemical and thermal properties of Mexican plum (Spondias purpurea L.). CyTA Journal of Food, 16(1), 127-134. doi:10.1080/19476337.2017.1345984

Nail, S. L., \& Gatlin, G. A. (1992). Freeze-drying: principles and practice. In: Avis, K. E., Lieberman, H. A., \& Lachman, L. (eds.), Pharmaceutical dosage forms: parenteral medications, Volume 2, Marcel Dekker Inc., New York. pp. 163-233.

Neldawati, Ratnawulan \& Gusnedi. (2013). Analysis of absorbance value in determining flavonoid levels for various types of leaves of medicinal plants. Pillar of Physics 2, 76-83.

Nimalaratne, C., \& Wu, J. (2015). Hen egg as an antioxidant food commodity: a review. Nutrients 7, 8274-8293. doi:10.3390/nu7105394

Nireesha, G. R., Divya, L., Sowmnya, C., Venkateshan, N., Babu, N., \& Lavakumar, V. (2013). Lyophilization/freeze-drying-an review. International Journal of Novel Trends in Pharmaceutical Sciences 3(4), 87-93. 
Osterlie, M., \& Lerfall, J. (2004). Lycopene from tomato products added minced meat: Effect on storage quality and color. Food Research International, 38, 925-929.

Pinela, J., Beatriz, M., Oliveira, P., \& Ferreira, I. (2016). Bioactive compounds of tomatoes as health promoters. Natural Bioactive Compounds from Fruits and Vegetables, 2, 48-91.

Polyakov, N., Foscan, A., Bowman, M., \& Kispert, L. (2006). Free radical formation in novel carotenoid metal ion complexes of astaxanthin. Journal of Physical Chemistry B 114(50), 1696816977.

Sambasevam, K., Mohamad, S., Sarih, N., \& Ismail, N. (2013). Synthesis and characterization of the inclusion complex of $\beta$ cyclodextrin and Azomethine. International Journal of Molecular Scinece 14(2), 3671-3682. doi:10.3390/ijms14023671

Santos, C. D., Buera, P., \& Mazzobre, F. (2017). Novel trends in cyclodextrins encapsulation: applications in food science. Current Opinion in Food Science, 16, 106-113.

Sethi, A., Sher, M., Akram, M.F., Karim, S., Khiljee, S., Sajjad, A., Shah, S. N. H., \& Murtaza, G. (2013). Albumin as a drug delivery and diagnostic tool and its market approved products. Acta Poloniae Pharmaceutica - Drug Research, 70(4), $597-$ 600.

Shah, R. S., Shah, R. R., Pawar. R. B., \& Gayakar, P. P. (2015). UVvisible spectroscopy - a review. International Journal of Institutional Pharmacy and Life Science, 5(5), 490-505.

Shi, J. (2000). Likopen in tomatoes: chemical and physical properties affected by food processing. Critical Reviews in Food Science and Nutrition, 40(1), 1-42.

Temitope, A., Eloho, A., \& Olubunmi, I. (2009). Lycopene content in tomatoes (Lycopersicon esculentum Mill): effect of thermal heat and its health benefits. Fresh Produce, 3(1), 40-43.

Wang, H. Y., Han, J., \& Feng, X. G. (2007). Spectroscopic study of orange G- $\beta$-cyclodextrin complex and its analytical application. Spectrochim. Acta Part A: Molecular and Biomolecular Spectroscopy, 66, 578-585. doi:10.1016/j.saa.2006.03.03

Wang, H., Wang, S., Zhu, H., Wang, S \& Xing, J. (2019). Inclusion complexes of lycopene and $\beta$-Cyclodextrin: preparation, characterization, stability and antioxidant activity. Antioxidants 8, 314. doi:10.3390/antiox 8080314 